\title{
Expression and clinical correlation of Survivin and PTEN in gastric cancer patients
}

\author{
AIKEREMU YUSUFU, ROUSIDAN TUERDI, DAREBAI REDATI, \\ AIZIMAITI REHEMUTULA, ZE-LIANG ZHAO and HAI-JIANG WANG \\ Department of Gastrointestinal Surgery, Tumor Hospital Affiliated to Xin Jiang Medical University, \\ Urumqi, Xinjiang Uygur Autonomous Region 830011, P.R. China
}

Received August 9, 2019; Accepted February 4, 2020

DOI: $10.3892 / \mathrm{ol} .2020 .12160$

\begin{abstract}
Reports on the correlation between the expression of Survivin/phosphatase and tensin homolog (PTEN) proteins and clinical factors in gastric cancer (GC) are varied, and the sample sizes were also not sufficient. The present study aimed to detect the expression of Survivin and PTEN proteins in GC patients on the basis of a greater number of specimens and to analyze the correlation with clinical features and survival. The results revealed that the Survivin expression rates in GC, normal tissues and metastatic lymph nodes were $72 \%(232 / 322), 5 \%(6 / 120)$ and $80 \%$ (36/45), respectively, while the PTEN expression rates were 34\% (109/322), $92.5 \%$ $(111 / 120)$ and $24.4 \%$ (11/45), respectively, and the differences between cancer and normal tissue or metastatic lymph nodes were significant for both proteins $(\mathrm{P}<0.05)$. The expression of Survivin was significantly associated with gross type, depth of invasion, distant metastasis, tumor, necrosis and metastasis (TNM) stage and vascular invasion, while PTEN expression was predominantly associated with age, tumor size, invasion depth, TNM stage and lymphatic invasion in GC patients $(\mathrm{P}<0.05)$. The expression of both was associated with postoperative metastasis and metastatic site $(\mathrm{P}=0.007$ and $\mathrm{P}=0.011$ for Survivin, and $\mathrm{P}=0.002$ and $\mathrm{P}=0.005$ for $\mathrm{PTEN}$ ). There was a negative association between the expression levels of Survivin and PTEN ( $\mathrm{P}=0.001, \mathrm{r}=-0.524)$. The expression levels of both were also associated with prognosis. The expression of Survivin and PTEN protein exhibit opposing trends in GC, which may indicate adverse biological effects in the occurrence of GC. The Survivin and PTEN expression levels are likely to be an important molecular event in gastric
\end{abstract}

Correspondence to: Dr Hai-Jiang Wang, Department of Gastrointestinal Surgery, Tumor Hospital Affiliated to Xin Jiang Medical University, 789 East Su Zhou Road, Urumqi, Xinjiang Uygur Autonomous Region 830011, P.R. China

E-mail: wanghaijiang731@126.com

Key words: gastric cancer, Survivin, PTEN, clinical feature, prognosis tumorigenesis and may be considered as molecular markers of GC progression and reliable prognostic indicators of GC.

\section{Introduction}

Gastric cancer (GC) comprises $>1,000,000$ new cases with an estimated 783,000 deaths reported worldwide in 2018, which renders it the fifth most common malignancy and the third primary cause of cancer-related mortality (1). GC also rates third in morbidity and second in mortality in China (2). The curative treatment for GC remains the complete excision of primary tumors with proper lymphadenectomy, since the curative effects of neoadjuvant therapy have been disappointing to date. Even some GC patients with the same TNM stage have a different prognosis and treatment outcome. Thus, researchers are focusing on identifying the molecular biomarkers and development-related targets of treatment. Treatment of the disease at an earlier stage may be key to improving the prognosis of patients with GC.

Survivin has been depicted as the smallest member of the 'inhibitor of apoptosis' family with a unique structure (3). Survivin is frequently observed to be overexpressed in malignancies when compared with normal tissues (4). As regards its functions, Survivin plays a regulatory role in cell division and the inhibition of apoptosis, induces angiogenesis, and plays a vital role in cancer progression (5). Survivin blocks apoptosis induced by a variety of pro-apoptotic stimuli, including chemotherapy and radiation, in numerous malignancies (6). In addition, an increased level of Survivin is correlated with a poorer outcome in various malignancies (4,7-10); however, certain studies have indicated that an increased expression of Survivin splice variants may represent a favorable marker of survival in some malignancies $(11,12)$.

Phosphatase and tensin homologue deleted on chromosome 10 (PTEN), also known as mutated in multiple advanced cancers (MMAC), was identified in 1997 by two independent research groups as a candidate tumor suppressor gene that was located at the human chromosome $10 \mathrm{q} 23$, a site frequently damaged in primary human malignancies $(13,14)$. The loss of PTEN function can occur via a series of genetic or epigenetic abnormalities, such as point chromosomal deletions, mutations, promoter hypermethylation and post-translational modifications $(15,16)$, and may result in various human malignancies, 
including renal cancer (17), pancreatic cancer (18), glioma (19), colorectal cancer (20), breast cancer (21), endometrial cancer (22), melanoma (23) and myeloid malignancies (24).

Although previous studies have reported enhanced expression of Survivin and a decreased expression of PTEN in GC, the level and correlation of Survivin and PTEN variations have not yet been fully elucidated. Although some studies have depicted their expression patterns in GC and analyzed the association of these expression patterns with clinical characteristics and prognosis $(25,26)$, the outcomes have been controversial or dubious due to the insufficiency of sample sizes $(27,28)$. Therefore, on the base of a relatively larger sample size, the present study further investigated Survivin and PTEN expression in GC in order to determine their expression levels, their effects on patient survival and clinical significance in GC.

\section{Materials and methods}

GC patients and specimens. A total of 322 primary gastric adenocarcinoma samples, 120 matched normal controls (situated $15 \mathrm{~cm}$ from the tumor margin) and 45 metastatic lymph nodes, which had been selected from the Tumor Hospital Affiliated to Xin Jiang Medical University between January, 2009 and December, 2012, were included in this study. Patient characteristics are shown in Table I. The study protocol was approved by the Review Board of Xin Jiang Tumor Hospital of Xin Jiang Medical University (approval no. 20090102, January 2, 2009) and all procedures followed the principles of the Declaration of Helsinki. All subjects provided written informed consent prior to participation. All patients had undergone radical primary tumor excision. All patients, apart from those with stage IV disease, underwent radical surgery (D2) followed by standard chemotherapy. None of the patients had received preoperative chemotherapy or radiotherapy. All cases were staged in accordance with the guidelines of the American Joint Committee on Cancer and the 2010 Cancer Staging manual of the Union for International Cancer Control (25). The data on clinical follow-up were obtained from the hospital record department. The overall survival (OS) time was calculated from the date of primary surgery to the date of death. The disease-free survival (DFS) time was measured from the date of primary radical surgery to the date of onset of local recurrence or distant metastasis. The patients who died due to surgery or other causes were eliminated from this study.

Immunohistochemistry (IHC). The streptavidin-biotin peroxidase complex (SP) method was used for the IHC assay and was completed with a commercially available SP-kit (SP-9000; OriGene Technologies, Inc.). All tumor specimens that were embedded in paraffin were cut into $4-\mu \mathrm{m}$ sections and rehydrated in a gradient series of alcohols following deparaffinization. The slides were boiled for antigen retrieval in $10 \mathrm{mM}$ sodium citrate buffer ( $\mathrm{pH} \mathrm{6.0)}$ and quenched with $3 \% \mathrm{H}_{2} \mathrm{O}_{2}$ for 15 min to block endogenous peroxidase activity. Non-specific binding was prevented by incubating the slides with 5\% goat serum (OriGene Technologies, Inc.) in PBS for $30 \mathrm{~min}$ at room temperature. The slides were treated with rabbit polyclonal anti-human Survivin (cat. no. RAB-0536; NeoMarkers, Inc.) antibody (1:100) (ready to use) or mouse monoclonal anti-human PTEN (cat. no. 17A; NeoMarkers, Inc.) antibody (ready to use, 1:50) at room temperature for $2 \mathrm{~h}$, followed by incubation with biotinylated goat anti-rabbit IgG (HRP; 1:10,000; cat. no. ab6721) and streptavidin-peroxidase (cat. no. TS-060-HR; Thermo Fisher Scientific, Inc.) for $1 \mathrm{~h}$ at room temperature. Diaminobenzidine was used for visualizing the peroxidase binding at room temperature for $1 \mathrm{~h}$. The slides were counterstained lightly with hematoxylin at room temperature for $1 \mathrm{~h}$ and visualized under an light microscope (magnification, x100). PBS was employed as a negative control in the IHC assay.

Evaluation of IHC staining. A tumor cell cytoplasm that stained brown under a light microscope was considered as positive staining. The cells were scored grossly based on the intensity of staining and the percentage of positive tumor cells (26). The intensity of staining (I) was scored as follows: The absence of staining as 0 points; weak staining as 1 point; and moderate to strong staining as 2 points. The percentage of positive tumor cells $(\mathrm{P})$ was divided into three grades as follows: None or $<10 \%$ of tumor cells with positive staining as 0 points; $10-50 \%$ as 1 point; and $>50 \%$ as 2 points. The total score was calculated as (I) $x(P)$ and the outcome was graded as $0,1+, 2+$ or $3+$. All stained slides were scored independently by two individual pathologists. The evaluation was performed twice, with the evaluator having no knowledge of the patient's diagnosis or prognosis. A total of two pathologists jointly examined the cases and came to an agreement towards any inconsistent results of the samples.

Statistical analysis. The SPSS software package (version 18.0 SPSS Inc.) was used for correlation analysis between categorical variables. The difference in the clinical features between the positive and negative groups was assessed using the Chi-squared or Fisher's exact tests. The patient OS and DFS were calculated using the Kaplan-Meier method. Univeriate and multivariate analysis was performed to analyze the factors that were determined to be significant for OS by the Cox proportional hazards model. All experiment were performed in triplicate. $\mathrm{P}<0.05$ was considered to indicate a statistically significant difference.

\section{Results}

General data of the samples. Among the 322 cases examined, there were 206 (63.9\%) males and 116 (36.1\%) females, with a mean age of 62 years (range, 25 to 87 years). A total of $296(91.9 \%)$ cases were categorized as differentiated type and $26(8.1 \%)$ as undifferentiated type (including mucinous adenocarcinoma and signet-ring cell carcinoma;). The invasion depth was T1 in 14 (4.3\%), T2 in 54 (16.8\%), T3 in 133 (41.3\%) and T4 in 121 (37.6\%) patients. As regards TNM staging, 31 patients had stage I disease, 78 had stage II, 159 had stage III and 54 had stage IV disease. In total, 212 cases exhibited regional lymph node metastasis. The follow-up data were complete in 292 cases and the median duration of follow-up was 38 months (range, 8-110 months) after primary 
Table I. Survivin and PTEN expression in gastric cancer and normal tissues.

\begin{tabular}{|c|c|c|c|c|c|c|c|}
\hline \multirow[b]{2}{*}{ Tissue } & \multirow[b]{2}{*}{$\mathrm{N}$} & \multicolumn{2}{|c|}{ Survivin } & \multirow[b]{2}{*}{$\mathrm{P}$-value } & \multicolumn{2}{|c|}{ PTEN } & \multirow[b]{2}{*}{ P-value } \\
\hline & & - & + & & - & + & \\
\hline GC tissue & 322 & $90(28)$ & $232(72)$ & $<0.001^{\mathrm{a}}$ & $213(66)$ & $109(34)$ & $<0.001^{\mathrm{a}}$ \\
\hline ANT & 120 & $114(95)$ & $6(5)$ & $<0.001^{\mathrm{b}}$ & $9(7.5)$ & $111(92.5)$ & $<0.001^{\mathrm{b}}$ \\
\hline LNM & 45 & $9(4.2)$ & $36(13.1)$ & $0.208^{c}$ & $34(75.6)$ & $11(24.4)$ & $0.102^{\mathrm{c}}$ \\
\hline
\end{tabular}

${ }^{a}$ comparison between GC and ANT. ${ }^{b}$ comparison between GC and LNM. ${ }^{c}$ comparison between ANT and LNM. ANT, adjacent normal tissue; LNM, lymph node metastasis; GC, gastric cancer; PTEN, phosphatase and tensin homolog.
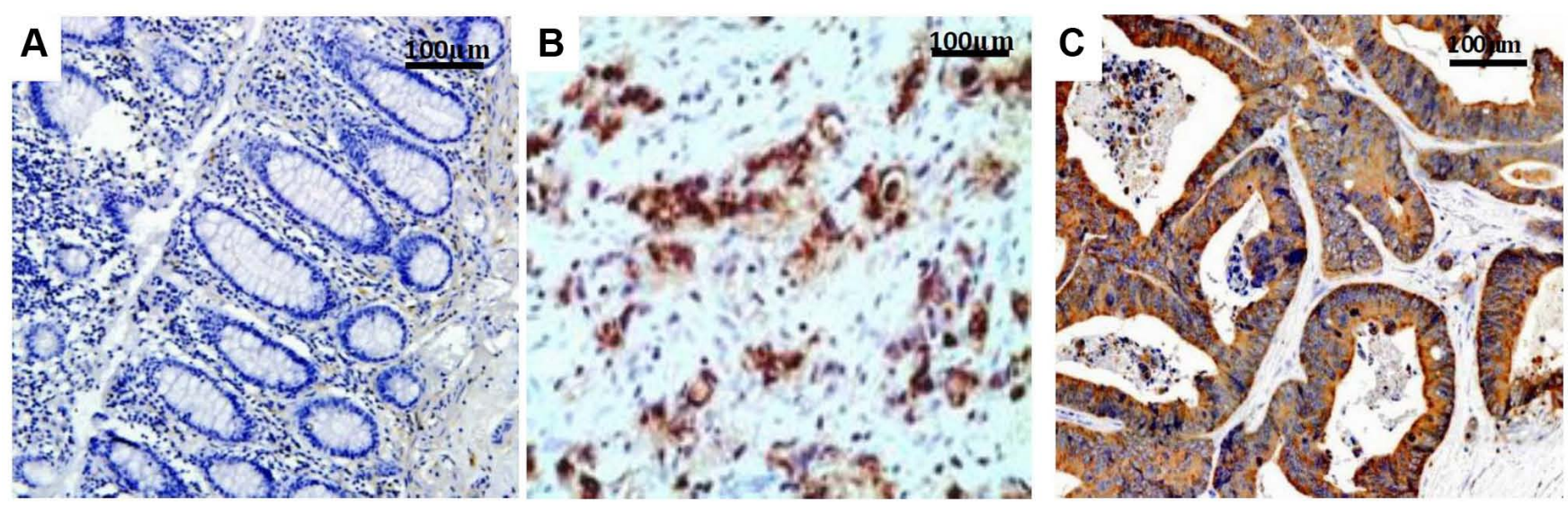

Figure 1. Survivin expression. (A) GC tissue, (B) Adjacent normal tissue and (C) metastatic lymph node. The staining level demonstrated gradual increase in the sequence of GC tissue, adjacent normal tissue and metastatic lymph node. GC, gastric cancer.
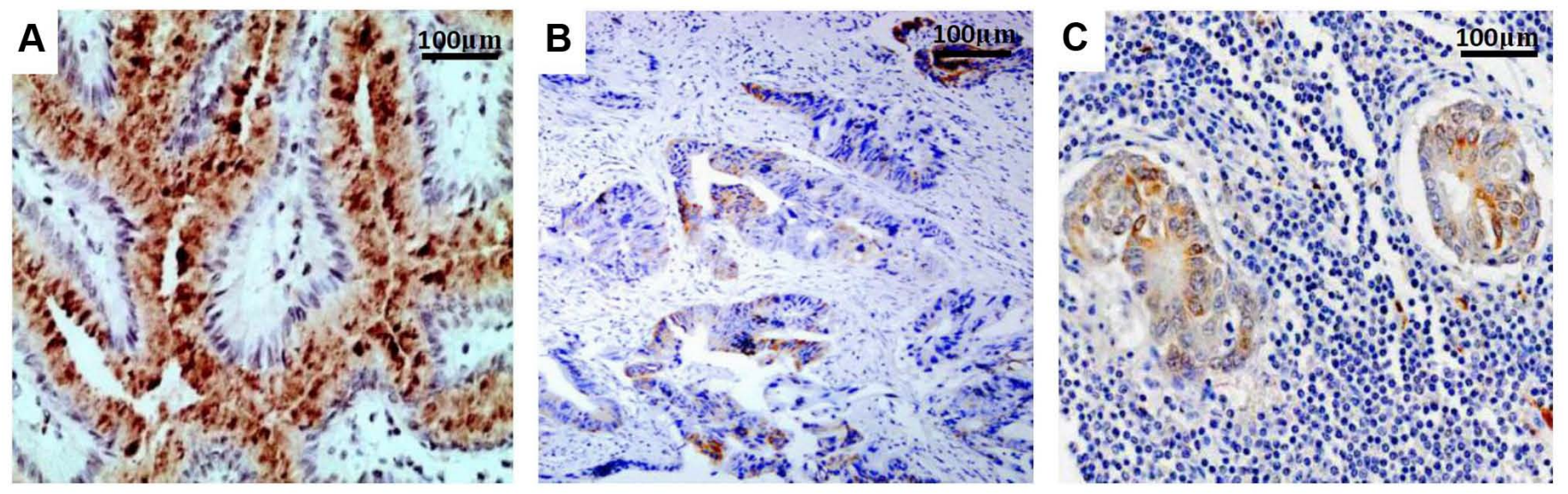

Figure 2. PTEN expression. (A) GC tissue, (B) Adjacent normal tissue and (C) metastatic lymph node. The staining level showed a gradual decreasing trend in the throughout the GC tissue, adjacent normal tissue and metastatic lymph node, respectively. GC, gastric cancer; PTEN, phosphatase and tensin homolog.

surgery. A total of 30 patients were lost during follow-up. The follow-up rate was $90.67 \%$.

Expression of Survivin or PTEN in GC, normal tissues and metastatic lymph nodes. Survivin expression was located mainly in the cytoplasm and cell membrane, with minimal expression in the nucleus (Fig. 1), while PTEN expression was observed in both the cytoplasm and nucleus (Fig. 2). The Survivin expression rates in the GC, normal tissues and metastatic lymph nodes were $72 \%$ (232/322), 5\% (6/120) and $80 \%$ (36/45), respectively (Fig. 1), while the PTEN expression rates were $34 \%$ (109/322), $92.5 \%$ (111/120) and $24.4 \%$ (11/45), respectively (Table I). The primary GC tissues and metastatic lymph nodes expressed significantly increased levels of Survivin and decreased levels of PTEN compared with the normal mucosal tissues $(\mathrm{P}<0.001)$. However, the difference in Survivin or PTEN expression between the GC tissues and metastatic lymph nodes was not statistically 
Table II. The correlation between Survivin and PTEN expression and clinicopathological factors.

\begin{tabular}{|c|c|c|c|c|c|c|c|}
\hline \multirow[b]{2}{*}{ Clinicopathological factors } & \multirow[b]{2}{*}{$\mathrm{N}=322$} & \multicolumn{3}{|c|}{ PTEN expression } & \multicolumn{3}{|c|}{ Survivin expression } \\
\hline & & $(-) \mathrm{n}(\%)$ & $(+) \mathrm{n}(\%)$ & P-value & $(-) \mathrm{n}(\%)$ & $(+) \mathrm{n}(\%)$ & P-value \\
\hline \multirow[t]{2}{*}{ Age } & $\leq 60$ & $34(36.6)$ & $109(47.6)$ & 0.071 & $102(49)$ & $41(36)$ & $0.024^{\mathrm{a}}$ \\
\hline & $>60$ & $59(63.4)$ & $120(52.4)$ & & $106(51)$ & $73(64)$ & \\
\hline \multirow[t]{2}{*}{ Sex } & Male & $58(62.4)$ & $148(64.6)$ & 0.701 & $131(63)$ & $75(65.8)$ & 0.616 \\
\hline & Female & $35(37.6)$ & $81(35.4)$ & 0.846 & 77 (37) & $39(34.2)$ & \\
\hline \multirow[t]{2}{*}{ Race } & Han & $89(95.7)$ & $218(95.2)$ & & $196(94.2)$ & $111(97.4)$ & 0.201 \\
\hline & Uyghur & $4(4.3)$ & $11(4.8)$ & & $12(5.8)$ & $3(2.6)$ & \\
\hline \multirow[t]{4}{*}{ Blood type } & A & $32(34.4)$ & $80(34.8)$ & 0.404 & $75(36.1)$ & $37(32.5)$ & 0.813 \\
\hline & $\mathrm{B}$ & $37(39.8)$ & $78(34.1)$ & & $71(34.1)$ & $44(38.6)$ & \\
\hline & $\mathrm{AB}$ & $4(4.3)$ & $22(9.6)$ & & $18(8.7)$ & $8(7)$ & \\
\hline & $\mathrm{O}$ & $20(21.5)$ & $49(21.4)$ & & $44(21.2)$ & $25(21.9)$ & \\
\hline \multirow[t]{3}{*}{ Tumor size } & $\leq 4$ & $16(17.2)$ & $38(16.6)$ & 0.973 & $33(15.9)$ & $21(18.4)$ & $0.012^{\mathrm{a}}$ \\
\hline & $4-8$ & $65(69.9)$ & $163(71.2)$ & & $157(75.5)$ & $71(62.3)$ & \\
\hline & $>8$ & $12(12.9)$ & $28(12.2)$ & & $18(8.7)$ & $22(19.3)$ & \\
\hline \multirow[t]{3}{*}{ Gross type } & Fungus & $35(37.6)$ & $93(40.6)$ & $0.022^{\mathrm{a}}$ & $88(42.3)$ & $40(35.1)$ & 0.193 \\
\hline & Ulcerous & $38(40.9)$ & $113(49.3)$ & & 97 (46.6) & $54(47.4)$ & \\
\hline & Invasive & $18(21.4)$ & $23(10.0)$ & & $23(11.1)$ & $20(17.5)$ & \\
\hline \multirow[t]{6}{*}{ Histology } & Well & $18(19.4)$ & $35(15.3)$ & 0.883 & $27(13)$ & $26(22.8)$ & 0.208 \\
\hline & Moderate & $58(62.4)$ & $147(64.2)$ & & $142(68.3)$ & $63(55.3)$ & \\
\hline & Poor & $8(8.6)$ & $16(7)$ & & $14(6.7)$ & $10(8.8)$ & \\
\hline & ry adenocarcinoma & $3(3.2)$ & $11(4.8)$ & & $9(4.3)$ & $5(4.4)$ & \\
\hline & us adenocarcinoma & $5(5.4)$ & $17(7.4)$ & & $13(6.3)$ & $9(7.9)$ & \\
\hline & Signet ring & $1(1.1)$ & $3(1.3)$ & & $3(1.4)$ & $1(0.9)$ & \\
\hline \multirow[t]{3}{*}{ Tumor site } & Antrum & $27(29)$ & $77(33.6)$ & 0.668 & $67(32.2)$ & $37(32.5)$ & 0.884 \\
\hline & Body & $28(30.1)$ & $69(30.1)$ & & $61(29.3)$ & $36(31.6)$ & \\
\hline & GEJ & $38(40.9)$ & $83(36.2)$ & & $80(38.5)$ & $41(36)$ & \\
\hline \multirow[t]{4}{*}{ Infiltration depth } & $\mathrm{T} 1$ & $5(5.4)$ & $9(3.9)$ & $<0.001^{\mathrm{a}}$ & $5(2.4)$ & $9(7.9)$ & $0.001^{\mathrm{a}}$ \\
\hline & $\mathrm{T} 2$ & $28(30.1)$ & $26(11.4)$ & & $28(13.5)$ & $26(22.8)$ & \\
\hline & $\mathrm{T} 3$ & $44(47.3)$ & 89 (38.9) & & $83(39.9)$ & $50(43.9)$ & \\
\hline & $\mathrm{T} 4$ & $16(17.2)$ & $105(45.9)$ & & $92(44.2)$ & $29(25.4)$ & \\
\hline \multirow[t]{3}{*}{ LN metastasis } & None & $37(39.8)$ & $73(31.9)$ & 0.206 & $60(28.8)$ & $50(43.9)$ & $0.025^{\mathrm{a}}$ \\
\hline & $\leq 7$ & $39(41.9)$ & $121(52.8)$ & & $117(56.3)$ & $43(37.7)$ & \\
\hline & $>7$ & $17(18.3)$ & $35(15.3)$ & & $31(14.9)$ & $21(18.4)$ & \\
\hline \multirow[t]{2}{*}{ Distant metastasis } & No & $86(92.5)$ & $182(79.5)$ & $0.005^{\mathrm{a}}$ & $169(81.3)$ & $99(86.8)$ & 0.199 \\
\hline & Yes & $7(7.5)$ & $47(20.5)$ & & $39(18.8)$ & $15(13.2)$ & \\
\hline \multirow[t]{4}{*}{ TNM staging } & I & $22(23.7)$ & $9(3.9)$ & $<0.001^{\mathrm{a}}$ & $7(3.4)$ & $24(21.1)$ & $<0.001^{\mathrm{a}}$ \\
\hline & II & $15(16.1)$ & $63(27.5)$ & & $53(25.5)$ & $25(21.9)$ & \\
\hline & III & $49(52.7)$ & $110(48)$ & & $109(52.4)$ & $50(43.9)$ & \\
\hline & IV & $7(7.5)$ & $47(20.5)$ & & $39(18.8)$ & $15(13.2)$ & \\
\hline \multirow[t]{2}{*}{ Vascular invasion } & No & $75(84.3)$ & $214(91.8)$ & 0.045 & $195(86.7)$ & $77(79.4)$ & 0.098 \\
\hline & Yes & $14(14.8)$ & $19(8.2)$ & & $30(13.3)$ & $20(20.6)$ & \\
\hline \multirow[t]{2}{*}{ Lymphatic invasion } & No & $101(88.6)$ & $171(82.2)$ & 0.130 & $201(87.4)$ & $71(77.2)$ & 0.022 \\
\hline & Yes & $13(11.4)$ & $37(17.8)$ & & $29(12.6)$ & $21(22.8)$ & \\
\hline \multirow[t]{2}{*}{ CEA } & $\leq 3.5$ & $18(20)$ & $58(27.9)$ & 0.152 & $52(27.4)$ & $24(22.2)$ & 0.327 \\
\hline & $>3.5$ & $72(80)$ & $150(72.1)$ & & $138(72.6)$ & $84(77.8)$ & \\
\hline
\end{tabular}

${ }^{\text {a }}<<0.05$. GEJ, gastroesophageal junction; LN, lymph node; CEA, carcinoembryonic antigen; PTEN, phosphatase and tensin homolog.

significant $(\mathrm{P}=0.208$ for Survivin and $\mathrm{P}=0.102$ for $\mathrm{PTEN})$, although Survivin expression was slightly increased and
PTEN expression was slightly decreased in the metastatic lymph nodes compared with in the cancerous tissues. Among 
Table III. Correlation between Survivin or PTEN expression and related factors of prognosis.

\begin{tabular}{|c|c|c|c|c|c|c|}
\hline \multirow[b]{2}{*}{ Prognostic factors } & \multicolumn{3}{|c|}{ Survivin } & \multicolumn{3}{|c|}{ PTEN } \\
\hline & $-\mathrm{N}(\%)$ & $+\mathrm{N}(\%)$ & P-value & $-\mathrm{N}(\%)$ & $+\mathrm{N}(\%)$ & P-value \\
\hline \multicolumn{7}{|l|}{ Local recurrence } \\
\hline No & $74(88.1)$ & $195(90.3)$ & 0.577 & $184(88.5)$ & $105(92.1)$ & 0.303 \\
\hline Yes & $10(11.9)$ & $21(9.7)$ & & $24(11.5)$ & $9(7.9)$ & \\
\hline \multicolumn{7}{|c|}{ Postoperative metastasis } \\
\hline No & $62(72.1)$ & $100(54.9)$ & 0.007 & $90(53.3)$ & $72(72.7)$ & 0.002 \\
\hline Yes & $24(27.9)$ & $82(45.1)$ & & 79 (46.7) & $27(27.3)$ & \\
\hline \multicolumn{7}{|l|}{ Metastatic site } \\
\hline No & $61(70.9)$ & $100(54.9)$ & 0.011 & $89(52.7)$ & $72(72.7)$ & 0.005 \\
\hline Single & $13(5.1)$ & $59(32.4)$ & & $55(32.5)$ & $17(17.2)$ & \\
\hline PD & $12(14)$ & $23(12.7)$ & & $25(14.8)$ & $10(10.1)$ & \\
\hline Median DFS & 61 & 31 & $<0.001$ & 31 & 70 & $<0.001$ \\
\hline Median OS & 73 & 39 & $<0.001$ & 37 & 71 & $<0.001$ \\
\hline
\end{tabular}

OS, overall survival; DFS, disease-free survival; PTEN, phosphatase and tensin homolog.

Table IV. Relation between Survivin and PTEN expression.

\begin{tabular}{|c|c|c|c|c|}
\hline \multirow[b]{2}{*}{ Factor } & \multicolumn{2}{|c|}{ PTEN } & \multirow[b]{2}{*}{ P-value } & \multirow[b]{2}{*}{$r$} \\
\hline & + & - & & \\
\hline \multicolumn{5}{|c|}{ Survivin } \\
\hline+ & 44 & 185 & $<0.001$ & -0.531 \\
\hline- & 70 & 23 & & \\
\hline
\end{tabular}

PTEN, phosphatase and tensin homolog.

the 236 tissues with positive Survivin expression, there were 75 cases with weak staining (32.3\%), 97 cases with moderate staining (41.8\%) and 64 with strong staining (27.6\%). Among the 109 tissues with positive PTEN expression, 44 exhibited weak staining (40.4\%), 35 demonstrated moderate staining $(32.1 \%)$ and 30 displayed strong staining (27.5\%).

Association between the expression of Survivin or PTEN and clinical factors. The expression of Survivin was significantly associated with gross type, depth of invasion, distant metastasis, TNM stage and vascular invasion, while PTEN expression was predominantly associated with age, tumor size, invasion depth, TNM stage and lymphatic invasion in patients with $\mathrm{GC}(\mathrm{P}<0.05$; Table II). The expression of both was associated with postoperative metastasis and metastatic site $(\mathrm{P}=0.007$ and $\mathrm{P}=0.011$ for Survivin, and $\mathrm{P}=0.002$ and $\mathrm{P}=0.005$ for $\mathrm{PTEN}$ ) (Table III). Correlation analysis also revealed a negative correlation between Survivin and PTEN expression ( $\mathrm{P}=0.001, r=-0.531$, Table IV); the higher the expression of Survivin, the lower the expression of PTEN.

Survival analysis. The results of survival analysis by the Kaplan-Meier method are presented in Fig. 3. The 1-,
3- and 5-year survival rates of all patients were 91.9, 53.8 and $27.7 \%$, respectively. A statistically significant difference was observed in OS or DFS between the Survivin- or PTEN-positive and -negative patients $(\mathrm{P}<0.001$ and $\mathrm{P}=0.001)$. The patients who were Survivin ${ }^{+}$or $\mathrm{PTEN}^{-}$had lower OS and DFS compared with those who were Survivin ${ }^{-}$or PTEN ${ }^{+}$. Multivariate analysis using the Cox model was performed to analyze relevant prognostic factors in GC patients. The survival rate of the patients was significantly associated with invasion depth, advanced TNM stage, postoperative metastasis, tumor size and the Survivin and PTEN expression level $(\mathrm{P}<0.05$; Table $\mathrm{V})$. The OS and DFS of the patients who were simultaneously Survivin ${ }^{+}$and PTEN $^{-}$were the lowest compared to the others (patients with Survivin+ and PTEN+ or Survivin- and PTEN- or Survivin- and PTEN+) $(\mathrm{P}=0.001$ and $\mathrm{P}=0.001$ ).

\section{Discussion}

Survivin is observed in a number of human malignancies but is almost undetectable in normal tissues. Survivin expression is associated with a diminished apoptotic index, a poorer survival rate and an increased recurrence risk in the majority of tumors (29-31). Data on the expression of Survivin in different gastric tissues are limited, particularly in large sample analyses. Bury et al (32) reported that the Survivin expression rate was $73.17 \%$ in GC patients and Gu et al (25) found that Survivin was expressed at a rate of $62.9 \%(44 / 70)$ in GC tissues and $0 \%(0 / 20)$ in adjacent normal tissues; both studies had small sample sizes. In the present study, the expression rate of Survivin was $72 \%$ in malignant tissues, $5 \%$ in adjacent normal tissues and $80 \%$ in metastatic lymph nodes. Survivin expression was increased in malignant tissues and the expression in metastatic lymph nodes was the highest. These findings suggested that the upregulation of Survivin may be closely associated with malignant transformation and the invasive behavior of GC. 
Table V. Cox regression model for univariate and multivariate analyses of prognostic factors in gastric cancer.

\begin{tabular}{lcccc}
\hline Prognostic factor & Univariate HR $(95 \% \mathrm{CI})$ & P-value & Multivariate HR (95\% CI) & P-value \\
\hline Age & $0.824(0.619-1.097)$ & 0.184 & $1.436(0.600-3.435)$ & 0.416 \\
Sex & $0.995(0.742-1.334)$ & 0.975 & $1.108(0.786-1.562)$ & 0.557 \\
Ethnicity & $0.580(0.314-1.069)$ & 0.081 & $1.623(0.728-4.121)$ & 0.473 \\
Blood type & $0.805(0.543-1.193)$ & 0.28 & $0.814(0.522-1.269)$ & 0.363 \\
Tumor site & $1.066(0.900-1.264)$ & 0.459 & $0.723(0.329-1.847)$ & 0.513 \\
Histological type & $0.799(0.629-1.016)$ & 0.067 & $0.831(0.603-1.145)$ & 0.258 \\
Gross type & $1.109(0.706-1.743)$ & 0.652 & $0.780(0.465-1.309)$ & 0.347 \\
Tumor size & $0.994(0.741-1.203)$ & 0.054 & $1.426(1.091-1.862)$ & $0.009^{\mathrm{a}}$ \\
Lymphatic invasion & $1.241(0.934-2.623)$ & $0.042^{\mathrm{a}}$ & $0.582(0.124-1.690)$ & 0.164 \\
Vascular invasion & $2.251(1.283-3.421)$ & 0.084 & $1.324(0.452-4.378)$ & 0.87 \\
Invasion depth & $1.852(1.532-2.237)$ & $0.001^{\mathrm{a}}$ & $1.383(1.130-1.693)$ & $0.002^{\mathrm{a}}$ \\
LN metastasis & $1.527(1.267-1.840)$ & $0.021^{\mathrm{a}}$ & $0.589(0.402-0.970)$ & 0.098 \\
Distant metastasis & $2.726(1.959-3.792)$ & $0.002^{\mathrm{a}}$ & $0.460(0.218-0.973)$ & 0.102 \\
TNM Staging & $2.089(1.746-2.499)$ & $0.001^{\mathrm{a}}$ & $1.988(1.581-2.502)$ & $0.001^{\mathrm{a}}$ \\
Local recurrence & $1.227(0.786-1.918)$ & 0.368 & $0.824(0.481-1.411)$ & 0.481 \\
Postoperative metastasis & $2.933(2.178-3.950)$ & $0.001^{\mathrm{a}}$ & $1.561(1.100-2.215)$ & $0.013^{\mathrm{a}}$ \\
Metastatic site & $1.616(1.359-1.927)$ & $0.001^{\mathrm{a}}$ & $0.672(0.429-1.054)$ & 0.083 \\
PTEN & $0.219(0.158-0.304)$ & $0.001^{\mathrm{a}}$ & $0.228(0.157-0.332)$ & $0.001^{\mathrm{a}}$ \\
Survivin & $5.889(4.020-8.626)$ & $0.001^{\mathrm{a}}$ & $4.514(2.964-6.876)$ & $0.001^{\mathrm{a}}$ \\
CEA & $0.774(0.555-1.080)$ & 0.14 & $0.933(0.558-1.546)$ & 0.231 \\
\hline
\end{tabular}

${ }^{\text {a }} \mathrm{P}<0.05$. LN, lymph node; PTEN, phosphate and tensin homolog; CEA, carcinoembryonic antigen.

The association between the expression of Survivin and invasion depth or metastatic lymph node in GC remains unclear. Lins et al (27), reported that Survivin expression was not associated with the depth of invasion, lymph node metastasis or differentiation. However, Gu et al (25) demonstrated that Survivin expression was associated with tumor differentiation, depth of invasion and lymph node metastasis. The data of the present study also indicated that the expression of Survivin was associated with gross type, invasion depth, distant metastasis, TNM stage, vascular invasion, postoperative metastasis and metastatic site. Wang et al (33), concluded that a positive Survivin expression in the nuclei was associated with prognosis, although its positive expression in the cytoplasm was not associated with prognosis in GC. Chen et al (34), found that the upregulation of Survivin was associated with a worse survival rate in GC in 11 studies compared with the normal group. The results of the present study also supported the hypothesis that patients with positive Survivin expression exhibit shorter OS and DFS compared with those with negative Survivin expression.

There is increasing evidence to indicate that PTEN is a pivotal element that participates in the process of cancer development and progression (35), and that it is a prognostic and predictive biomarker in cancer (36). Li et al (37) reported positive expression of PTEN in 41.2\% (47/114) of cases, while a reduction or loss of PTEN expression was detected in over half of GC cases $(58.7 \%, 67 / 114)$. Zhu et al (38), found that the percentage of PTEN expression in GC samples $(48 \%$, 77/159) was significantly lower compared with that in adjacent normal tissue $(75 \%, 113 / 151)$. The present study demonstrated that PTEN expression in GC (34\%) and metastatic lymph nodes $(24.4 \%)$ was downregulated compared with in normal tissues $(92.5 \%)$. PTEN expression in metastatic lymph nodes was lower compared with that in GC tissues, although the difference did not reach statistical significance. Therefore, the loss or diminished expression of PTEN in malignant tissues of the stomach may be a major general event in the progression of GC.

Reports on the associations between PTEN expression and clinicopathological factors are inconsistent. Bai et al (39) demonstrated that age, differentiation, TNM classification, depth of invasion and distant metastasis were negatively associated with the expression of nuclear PTEN. Furthermore, the lower level of nuclear PTEN expression was also associated with a good prognosis. Li et al (37) found that the loss of PTEN expression was associated with distant metastasis and advanced clinical stage, but not with prognosis. The data of the present study demonstrated that PTEN expression was associated with age, tumor size, invasion depth, TNM stage, lymph node metastasis, lymphatic invasion, postoperative metastasis and metastatic site. Patients with positive PTEN expression had higher OS compared with those with negative PTEN expression. Therefore, low expression of PTEN may be a critical biomarker for GC progression and may be closely associated with cancer invasiveness and metastasis.

The correlation analysis in the present study demonstrated a negative correlation between Survivin and PTEN expression. Lu et al (40), demonstrated that the expression of PTEN was negatively correlated with the expression of Survivin in cervical intraepithelial neoplasia and cervical 
A
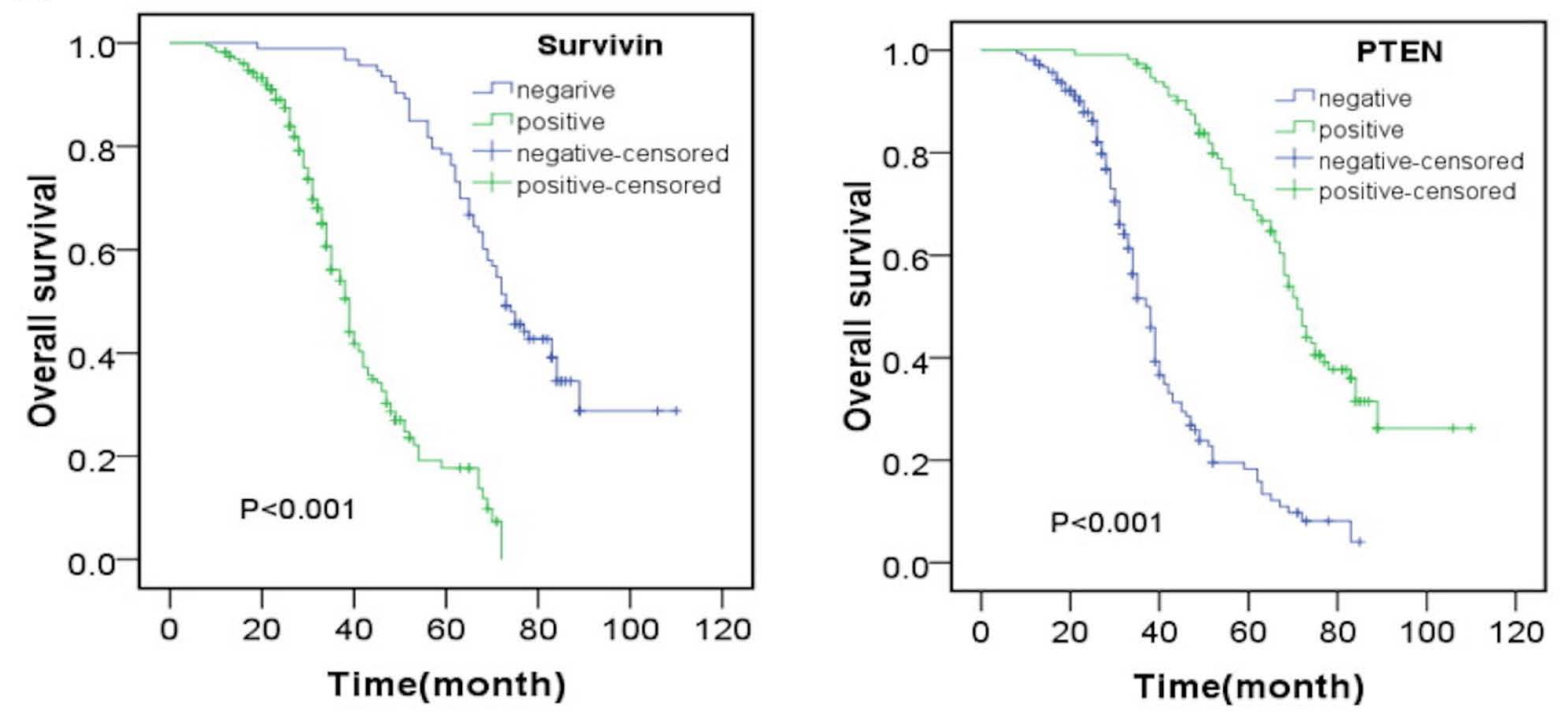

B
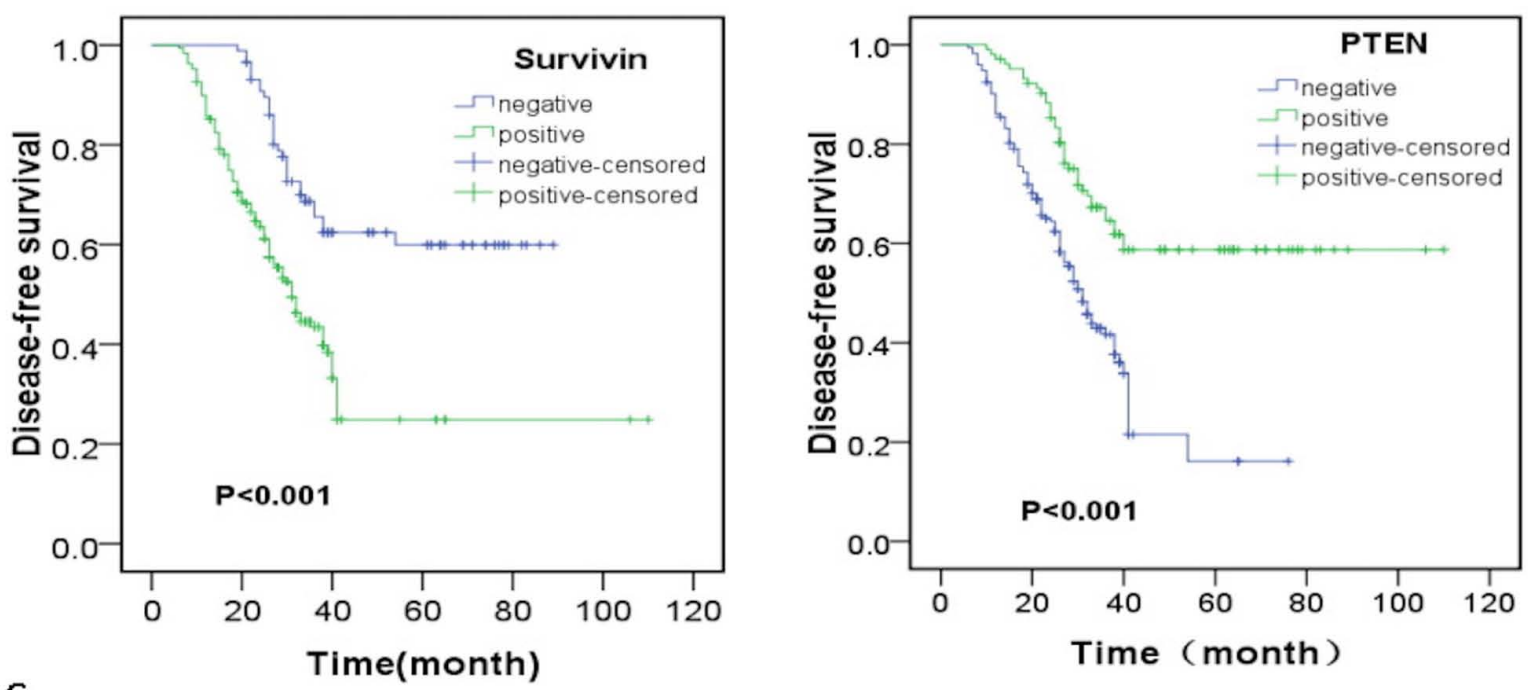

C

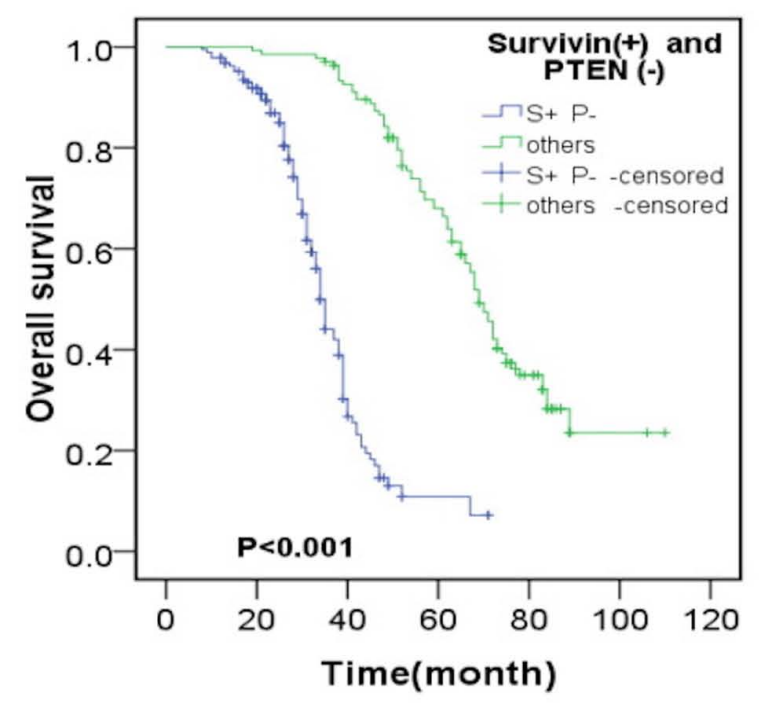

Figure 3. Correlation between expression level of Survivin or PTEN in GC and survival. (A) The difference in overall survival between Survivin or PTEN positive and negative patients. (B) The difference in disease free survival between Survivin or PTEN positive and negative patients. (C) The difference in overall survival between patients with simultaneous Survivin ${ }^{+}, \mathrm{PTEN}^{-}$and patients with others (patients with Survivin+and PTEN+ or Survivin- and PTEN- or Survivin- and PTEN+). GC, gastric cancer; PTEN, phosphatase and tensin homolog. 
squamous cell carcinoma. Wu et al (41), found that PTEN overexpression suppressed the growth of bladder cancer cells and significantly induced apoptosis via the downregulation of Survivin and caspase cascade activation. As previously demonstrated, following treatment with aspirin, Hep-2 cells exhibited a significant upregulation of PTEN and the inhibition of nuclear factor (NF)- $\mathrm{BB}$ and Survivin, the downstream targets of the PTEN/protein kinase B (AKT) signaling pathway, suggesting that the anticancer molecular mechanism of aspirin may be associated with the inhibition of tumor invasion and the induction of apoptosis by regulating the activity of the PTEN/AKT/NF- $\mathrm{KB} /$ Survivin signaling pathway (42). The aforementioned findings suggest that there may be an association between Survivin and PTEN.

In conclusion, Survivin and PTEN exhibit a negative association in GC, which may indicate that Survivin fuctions as an oncogene while PTEN plays the role of tumor suppressor gene in GC occurrence. PTEN and Survivin expression are likely important molecular events in gastric tumorigenesis and may be used as molecular markers of GC progression and reliable prognostic indicators of GC. The patient's odds of recurrence, metastasis and survival may be predicted preliminarily by detecting the Survivin and PTEN expression in GC tissue before treatment. The major limitation of the present study is that there wasn't the chance to perform the experiments in vitro and in vivo for further functional corroboration of the two genes, which is the main emphasis of future research.

\section{Acknowledgements}

Not applicable.

\section{Funding}

The present study was funded by the Natural Science Fund of the Xin Jiang Uyghur Autonomous Region (grant no. 2019D01C253).

\section{Availability of data and materials}

The datasets used and/or analyzed during the present study are available from the corresponding author upon reasonable request.

\section{Authors' contributions}

AY and HW conceived and designed the present study. RT acquired and analyzed the data and drafted the initial manuscript. DR assisted with the collection of the clinicopathological materials and the overall statistics. AR and ZZ helped perform the IHC experiment. All authors have read and approved the manuscript.

\section{Ethics approval and consent to participate}

The present study was approved by the Review Board of Xin Jiang Tumor Hospital of Xin Jiang Medical University (Urumqi, China; approval no. 20090102), and performed in accordance with The Declaration of Helsinki.
All subjects provided written informed consent prior to participation.

\section{Patient consent for publication}

Not applicable.

\section{Competing interests}

The authors declare that they have no competing interests.

\section{References}

1. Bray F, Ferlay J, Soerjomataram I, Siegel RL, Torre LA and Jemal A: Global Cancer Statistics 2018: GLOBOCAN estimates of incidence and mortality worldwide for 36 cancers in 185 countries. CA Cancer J Clin 68: 394-424, 2018.

2. Macdonald JS, Smalley SR, Benedetti J, Hundahl SA, Estes NC, Stemmermann GN, Haller DG, Ajani JA, Gunderson LL, Jessup JM and Martenson JA: Chemoradiotherapy after surgery compared with surgery alone for adenocarcinoma of the stomach or gastroesophageal junction. N Engl J Med 345: 725-730, 2001.

3. Ambrosini G, Adida C and Altieri DC: A novel anti-apoptosis gene, survivin, expressed in cancer and lymphoma. Nat Med 3: 917-921, 1997.

4. Margulis V, Lotan Y and Shariat SF: Survivin: A promising biomarker for detection and prognosis of bladder cancer. World J Urol 26: 59-65, 2008.

5. Pennati M, Folini $M$ and Zaffaroni N: Targeting survivin in cancer therapy. Expert Opin Ther Targets 12: 463-476, 2008.

6. Kato J, Kuwabara Y, Mitani M, Shinoda N, Sato A, Toyama T, Mitsui A, Nishiwaki T, Moriyama S, Kudo J and Fujii Y: Expression of survivin in esophageal cancer: Correlation with the prognosis and response to chemotherapy. Int J Cancer 95: 92-95, 2001.

7. Zhang LQ, Wang J, Jiang F, Xu L, Liu FY and Yin R: Prognostic value of survivin in patients with non-small cell lung carcinoma: A systematic review with meta-analysis. PLoS One 7: e34100, 2012.

8. Krieg A, Werner TA, Verde PE, Stoecklein NH and Knoefel WT: Prognostic and clinicopathological significance of survivin in colorectal cancer: A meta analysis. PLoS One 8: e65338, 2013.

9. Hingorani P, Dickman P, Garcia-Filion P, White-Collins A, Kolb EA and Azorsa DO: BIRC5 expression is a poor prognostic marker in Ewing sarcoma. Pediatr Blood Cancer 60: 35-40, 2013.

10. Carter BZ, Qiu Y, Huang X, Diao L, Zhang N, Coombes KR, Mak DH, Konopleva M, Cortes J, Kantarjian HM, et al: Survivin is highly expressed in CD34(+)38(-) leukemic stem/progenitor cells and predicts poor clinical outcomes in AML. Blood 120: 173-180, 2012

11. Vallböhmer D, Drebber U, Schneider PM, Baldus S, Bollschweiler E, Brabender J, Warnecke-Eberz U, Mönig S, Hölscher AH and Metzger R: Survivin expression in gastric cancer: Association with histomorphological response to neoadjuvant therapy and prognosis. J Surg Oncol 99: 409-413, 2009.

12. Jamieson NB, Carter CR, McKay CJ and Oien KA: Tissue biomarkers for prognosis in pancreatic ductal adenocarcinoma: A systematic review and meta-analysis. Clin Cancer Res 17: 3316-3331, 2011.

13. Steck PA, Pershouse MA, Jasser SA, Yung WK, Lin H, Ligon AH, Langford LA, Baumgard ML, Hattier T, Davis T, et al: Identification of a candidate tumour suppressor gene, MMAC1, at chromosome $10 \mathrm{q} 23.3$ that is mutated in multiple advanced cancers. Nat Genet 15: 356-362, 1997.

14. Ngeow J, Sesock K and Eng C: Clinical implications for germline PTEN spectrum disorders. Endocrinol Metab Clin North Am 46: 503-517, 2017.

15. Correia NC, Girio A, Antunes I, Martins LR and Barata JT: The multiple layers of non-genetic regulation of PTEN tumour suppressor activity. Eur J Cancer 50: 216-225, 2014.

16. Bermúdez Brito M, Goulielmaki E and Papakonstanti EA: Focus on PTEN regulation. Front Oncol 5: 166, 2015.

17. Que WC, Qiu HQ, Cheng Y, Liu MB and Wu CY: PTEN in kidney cancer: A review and meta-analysis. Clin Chim Acta 480: 92-98, 2018 
18. Hill R, Calvopina JH, Kim C, Wang Y, Dawson DW, Donahue TR, Dry S and Wu H: PTEN loss accelerates KrasG12D-induced pancreatic cancer development. Cancer Res 70: 7114-7124, 2010.

19. Han F, Hu R, Yang H, Liu J, Sui J, Xiang X, Wang F, Chu L and Song S: PTEN gene mutations correlate to poor prognosis in glioma patients: A meta-analysis. Onco Targets Ther 9: 3485-3492, 2016

20. Yazdani Y, Farazmandfar T, Azadeh H and Zekavatian Z: The prognostic effect of PTEN expression status in colorectal cancer development and evaluation of factors affecting it: Mir-21 and promoter methylation. J Biomed Sci 23: 9, 2016.

21. Golmohammadi R, Rakhshani MH, Moslem AR and Pejhan A: Prognostic role of PTEN gene expression and length of survival of breast cancer patients in the north east of Iran. Asian Pac J Cancer Prev 17: 305-309, 2016.

22. Zhang HM, Fan TT, Li W and Li XX: Expressions and significances of ttf-1 and PTEN in early endometrial cancer. Eur Rev Med Pharmacol Sci 21 (Suppl 3): S20-S26, 2017.

23. Giles KM, Rosenbaum BE, Berger M, Izsak A, Li Y,Illa Bochaca I, Vega-Saenz de Miera E, Wang J, Darvishian F, Zhong H and Osman I: Revisiting the clinical and biologic relevance of partial PTEN loss in melanoma. J Investig Dermatol 139: 430-438, 2019.

24. Morotti A, Panuzzo C, Crivellaro S, Carra G, Torti D, Guerrasio A and Saglio G: The role of PTEN inmyeloid malignancies. Hematol. Rep 7: 5844, 2015.

25. Gu Y, Jin S, Wang F, Hua Y, Yang L, Shu Y, Zhang Z and Guo R: Clinicopathological significance of PI3K, Akt and survivin expression in gastric cancer. Biomed Pharmacother 68: 471-475, 2014.

26. Yusup A, Huji B, Fang C, Wang F, Dadihan T, Wang HJ and Upur H: Expression of trefoil factors and TWIST1 in colorectal cancer and their correlation with metastatic potential and prognosis. World J Gastroenterol 23: 110-120, 2017.

27. Lins RR, Oshima CT, Oliveira LA, Silva MS, Mader AM and Waisberg J: Expression of E-cadherin and WNT pathway proteins Betacatenin, APC, TCF-4 and Survivin in gastric adenocarcinoma: Clinical and pathological implication. Arq Bras Cir Dig 29: 227-231, 2016.

28. Deng H, Wu RL, Zhou HY, Huang X, Chen Y and Liu LJ: Significance of Survivin and PTEN expression in full lymph node-examined gastric cancer. World J Gastroenterol 12: 1013-1017, 2006.

29. Contis J, Lykoudis PM, Goula K, Karandrea D and Kondi-Pafiti A: Survivin expression as an independent predictor of overall survival in pancreatic adenocarcinoma. J Cancer Res Ther 14 (Suppl): S719-S723, 2018.

30. Xia H, Chen S, Huang $\mathrm{H}$ and Ma H: Survivin over-expression is correlated with a poor prognosis in esophageal cancer patients. Clin Chim Acta 446: 82-85, 2015.

31. Li S, Wang L, Meng Y, Chang Y, Xu J and Zhang Q: Increased levels of LAPTM4B, VEGF and survivin are correlated with tumor progression and poor prognosis in breast cancer patients. Oncotarget 8: 41282-41293, 2017.
32. Bury J, Szumiło J, Dabrowski A, Ciechański A, Sliwińska J and Wallner G: Vascular endothelial growth factor and survivin immunostaining in gastric adenocarcinoma. Pol Przegl Chir 84: 341-347, 2012.

33. Wang ZN, Xu HM, Jiang L, Zhou X, Lu C and Zhang X: Expression of survivin in primary and metastatic gastric cancer cells obtained by laser capture microdissection. World J Gastroenterol 10: 3094-3098, 2004.

34. Chen J, Li T, Liu Q, Jiao H, Yang W, Liu X and Huo Z: Clinical and prognostic significance of HIF-1a, PTEN, CD44v6, and survivin for gastric cancer: A meta-analysis. PLoS One 9: e91842, 2014

35. Milella M, Falcone I, Conciatori F, Cesta Incani U, Del Curatolo A, Inzerilli N, Nuzzo CM, Vaccaro V, Vari S, Cognetti F and Ciuffreda L: PTEN: Multiple functions in human malignant tumors. Front Oncol 5: 24, 2015.

36. Bazzichetto C, Conciatori F, Pallocca M, Falcone I, Fanciulli M, Cognetti F, Milella M and Ciuffreda L: PTEN as a prognostic/predictive biomarker in cancer: An unfulfilled promise? Cancers (Basel) 11: 435, 2019.

37. Li Y, Cui J, Zhang CH, Yang DJ, Chen JH, Zan WH, Li B, Li Z and He YL: High-expression of DJ-1 and loss of PTEN associated with tumor metastasis and correlated with poor prognosis of gastric carcinoma. Int J Med Sci 10: 1689-1697, 2013.

38. Zhu X, Qin X, Fei M, Hou W, Greshock J, Bachman KE, Kang J and Qin Y: Loss and reduced expression of PTEN correlate with advanced-stage gastric carcinoma. Exp Ther Med 5: 57-64, 2013.

39. Bai ZG, Ye YJ, Shen DH, Lu YY, Zhang ZT and Wang S: PTEN expression and suppression of proliferation are associated with $\mathrm{Cdx} 2$ overexpression in gastric cancer cells. Int J Oncol 42 : 1682-1691, 2013

40. Lu D, Qian J, Yin X, Xiao Q, Wang C and Zeng Y: Expression of PTEN and survivin in cervical cancer: Promising biological markers for early diagnosis and prognostic evaluation. Brit J Biomed Sci 69: 143-146, 2012.

41. Wu ZX, Song TB, Li DM,Zhang XT and Wu XL: Overexpression of PTEN suppresses growth and induces apoptosis by inhibiting the expression of survivin in bladder cancer cells. Tumour Biol 28: 9-15, 2007.

42. Jin M, Li C, Zhang Q, Xing S, Kan X and Wang J: Effects of aspirin on proliferation, invasion and apoptosis of Hep-2 cells via the PTEN/AKT/NF- $\kappa \mathrm{B} /$ survivin signaling pathway. Oncol Lett 15: 8454-8460, 2018.

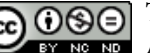

This work is licensed under a Creative Commons Attribution-NonCommercial-NoDerivatives 4.0 International (CC BY-NC-ND 4.0) License. 